\title{
PRIMEROS REGISTROS DE MÍSIDOS \\ (CRUSTACEA: PERACARIDA, MYSIDA) DEL PARQUE NACIONAL ARRECIFE PUERTO MORELOS, QUINTANA ROO, MÉXICO
}

\author{
Manuel Ortiz, Ignacio Winfield y Sergio Cházaro-Olvera
}

Laboratorio de crustáceos, Facultad de Estudios Superiores Iztacala,

Universidad Nacional Autónoma de México.ortiztouzet@yahoo.com.

\section{RESUMEN}

Los mísidos del Caribe mexicano permanecen poco estudiados hasta el presente. Este trabajo representa el primer registro de estos crustáceos para el Parque Nacional Arrecife Puerto Morelos, Quintana Roo, México. El material fue recolectado de macroalgas, esponjas y roca de coral, con ayuda de equipo SCUBA. Se documentan ocho especies de mísidos para dicho parque arrecifal. Las especies Heteromysis elegans, Brattegard, 1974; H. (Olivemysis) floridensis Brattegard, 1969 y H. (O.) siciliseta Brattegard, 1970, constituyen registros nuevos para el Caribe mexicano.

Palabras clave: Crustacea, Peracarida, Mysida, Caribe mexicano.

\section{FIRST RECORD OF MYSID SHRIMPS (CRUSTACEA, PERACARIDA, MYSIDA) FROM THE NATIONAL PARK CORAL REEF PUERTO MORELOS, QUINTANA ROO, MEXICO}

\section{ABSTRACT}

The Mysida of the mexican Caribbean are still very poor studied up today. This paper is the first contribution to the knowledge of the group of crustaceans in the Coral Reef National Park Puerto Morelos, Quintana Roo. The material was collected from different substrates with SCUBA, mainly sponges, macroalgae and coral rubble. A total of eight species of mysids were documented for this reef park. The species Heteromysis elegans, Brattegard, 1974; H. (Olivemysis) floridensis Brattegard, 1969 y H. (O.) siciliseta Brattegard, 1970, are new records for the Mexican Caribbean.

Keywords: Crustacea, Peracarida, Mysida, mexican Caribbean.

\section{INTRODUCCIÓN}

En la actualidad, existen tres órdenes de crustáceos peracáridos (Lophogastrida, Stygiomysida y Mysida) conocidos hasta hace unos años como misidáceos (Mysidacea) (Wittmann et al., 2014). De estos, los lofogástridos no suelen ser capturados en las aguas someras, los stigiomísidos son relativamente comunes en el ambiente troglobio, y los mísidos son los más comunes en el ambiente marino.

Muy poco se conoce sobre los mísidos del Caribe mexicano. El trabajo de Markham et al. (1990) representa la primera publicación sobre este grupo de crustáceos peracáridos. En el se citan las especies Heteromysis actiniae Clarke, 1955; H. disrupta Brattegard, 1970; H. mayana Brattegard, 1970; Mysidium columbiae (Zimmer, 1915); Siriella chierchiae Coifman, 1937; S. mexicana Brattegard, 1970 y Siriella sp. 
Más de una década después, Escobar-Briones (2002) cita 26 especies de mísidos para los ambientes salobres costeros y marinos mexicanos del Golfo de México: para la Laguna de Alvarado, Bowmaniella bacescui Brattegard, 1970 y Metamysidopsis swifti Băcescu, 1969; para Laguna de Tamiahua, Americamysis almyra (Bowman, 1954); para la Laguna Madre, Taphromysis bowmani Băcescu, 1961; para la Laguna de Términos, Americamysis almyra (Bowman, 1964), A. bahía (Molenock, 1969), Bowmaniella floridana Holmquist, 1975 = B. dissimilis (Coifmann, 1937), Brasilomysis castroi Băcescu, 1968, Mysidopsis badius Modlin, 1987, Siriella chierchiae, Coifman, 1937 y Thraphromysis villalobosi Escobar y Soto, 1988; para Tuxpan, B. brasiliensis Băcescu, 1968; para Veracruz, Bowmaniella dissimilis (Coifman, 1937). Además, se registra para el Caribe mexicano varias especies: para la Bahía Ascención, Amathimysis polita Brattegard, 1970, Diptromysis paucispinosa Brattegard, 1969 =Dioptromysis paucispinosa, Brattegard, 1969; para el Banco Chinchorro, Heteromysis actiniae Clarke, 1955, H. tuberculospina Modlin, 1987, Mysidium columbiae Zimmer, 1915; para la Isla Cozumel, H. bermudensis Sars, 1885, Mysidium integrum Tattersall, 1951 y para Majahual, Amathimysis cherados Brattegard, 1974, A. gibba Brattegard, 1969, Antromysis bahamensis (Brattegard, 1969) = Parvimysis bahamensis Brattegard, 1969, Mysidopsis velifera Brattegard, 1973 y Siriella mexicana Brattegard, 1970. Sin embargo, después de la revisión del género Bowmaniella, publicada por Heard y Price (2006), las 26 especies antes mencionadas quedan reducidas a 25, debido a que actualmente Bowmaniella bacescui Brattegard, 1970 es sinonimia de Coifmaniella jhonsoni (Tattersall, 1937) y B. floridana Holmquist, 1975 lo es de $B$. dissimilis (Coifmann, 1937). Finalmente, en la lista de Price y Heard (2009) sobre las 51 especies de mísidos del Golfo de México, se mencionan 12 especies para varias localidades caribeñas (ninguna para Puerto Morelos), incluyendo a Mysidopsis badius Modlin, 1987 para Belice.

Durante las colectas efectuadas por el laboratorio de crustáceos, de la Facultad de Estudios Superiores Iztacala, de la UNAM, en junio del 2013, fueron tomados 11552 peracáridos pertenecientes a los órdenes Amphipoda (5 466 especímenes), Isopoda (3 022), Tanaidacea (2 707), Cumacea (340) y Mysida (17).

\section{OBJETIVO}

-Hacer la determinación y ordenamiento taxonómicos de los mísidos colectados en el parque nacional Arrecife Puerto Morelos (PNAPM), Quintana Roo, México.

\section{MATERIALES Y MÉTODOS}

Área de estudio. El PNAPM se ubica en la costa del Caribe mexicano (20 $\left.51^{\prime} \mathrm{N}, 86^{\circ} 55^{\prime} \mathrm{O}\right)$ entre Cancún y Playa del Carmen, Quintana Roo. Este arrecife es una barrera de tipo bordeante con $21 \mathrm{~km}$ de largo, profundidades entre los 0.5 a $20 \mathrm{~m}$, y seis zonas estructurales: línea de costa, laguna arrecifal, arrecife posterior, cresta arrecifal, arrecife frontal y plataforma arenosa. El fondo de la laguna arrecifal está cubierta por una combinación de pastos marinos dominada por Thalassia testudinum, y la formación de montículos de roca coralina.

El trabajo de campo fue realizado en seis sitios de muestreo en el PNAPM, durante los días 4-10 de junio del 2013. La recolecta de los peracáridos se realizó mediante buceo autónomo (SCUBA) entre $\operatorname{los} 1$ a $\operatorname{los} 15 \mathrm{~m}$ de profundidad, en muestras de restos de coral, esponjas, fondos blandos, troncos, pastos marinos y macroalgas, de acuerdo a los permisos de colecta científica DGOPA.01024.110213.0236 y PPF/DGOPA-051/15. La recolecta y separación de los ejemplares en cada sustrato fue de acuerdo al protocolo propuesto 
por Winfield et al. (2013). Las muestras fueron identificadas en el laboratorio de crustáceos (FESIztacala-UNAM) de acuerdo a las claves específicas, ilustraciones y descripciones. El sistema de clasificación empleado es el propuesto por Wittmann et al. (2014). Los registros nuevos de mísidos para Puerto Morelos se señalan con un asterisco (*), mientras que los del Caribe mexicano con dos $(* *)$. La validez de todas las especies ha sido confirmada con WoRMS (2017).

\section{RESULTADOS}

La lista que aparece a continuación relaciona los táxones con sus autores y años para las aguas someras del parque nacional Arrecife Puerto Morelos. También se señala el sexo, el largo corporal, el sustrato y la fecha de colecta de cada especie.

Subphylum CRUSTACEA Brünnich, 1772

Subclase EUMALACOSTRACA Grobben, 1892

Superorden PERACARIDA Calman, 1904

Orden MYSIDA Boas, 1883

Familia MYSIDAE Haworth, 1825

Subfamilia SIRIELLINAE Czerniavsky, 1882

Tribu SIRIELLINI Czerniavsky, 1882

Género Siriella Dana, 1850

Siriella chierchie Coifman, 1937 (*); O̊ joven; 2 mm; roca de coral; 02/06/13.

Siriella mexicana Brattegard, 1970 (*); ô; $9.1 \mathrm{~mm}$; roca de coral; sobre Halimeda goreaui Taylor 1962; 02-06-13.

Subfamilia GASTROSACCINAE Norman, 1892

Tribu GASTROSACCINI Norman, 1892

Género Coifmaniella Heard y Price, 2006

Coifmaniella sp. (*) $9 ; 3 \mathrm{~mm}$; roca de coral; 04/06/07.

Subfamilia ERYTHROPINAE Hansen, 1910

Tribu ERYTHROPINI Hansen, 1910

Género Amathimysis Brattegard, 1969

Amathimysis gibba Brattegard, 1969 (*); + ovígera; 2.8 mm; sobre Halimeda goreaui; 05/06/13;

ō; 2.9 mm sobre H. goreaui; 10/06/13. 


\section{Subfamilia HETEROMYSINAE Norman, 1892 \\ Tribu HETEROMYSINI Norman, 1892}

Género Heteromysis Smith, 1873

Heteromysis (Olivemysis) bermudensis Sars, 1885 (*); ふૈ; $3.2 \mathrm{~mm}$; roca de coral; 07-06-13; con oostegitos; $4 \mathrm{~mm}$; roca de coral; 07/06/13.

Heteromysis elegans Brattegard, $1974\left(^{*}\right)(* *) ;$; 3 mm; roca de coral;

Heteromysis (Olivemysis) floridensis Brattegard, 1969 (*) (**); + con oostegitos; en esponja;

$3.7 \mathrm{~mm} ; 09-06-13$.

Heteromysis (Olivemysis) siciliseta Brattegard, $1970\left(^{*}\right)(* *) ;$ \% $; 4 \mathrm{~mm}$; roca de coral; $10 / 06 / 13$.

\section{CONCLUSIONES}

Al estudiar las 17 especies de mísidos recolectadas se han logrado identificar un total de cuatro subfamilias, tres tribus, cuatro géneros y 8 especies. La roca de coral resultó el sustrato con la mayor presencia de mísidos.

Se denota la ausencia de Anchialina typica (Kroyer, 1861), la cual junto a Siriella chierchiae, Coifman, 1937, constituyen las dos especies más abundantes en cualquier colecta del grupo en la región caribeña. Probablemente el sistema de muestreo empleado no fue el más indicado para este tipo de peracáridos. Además, los representantes del género Mysidium, asiduos visitantes de los sistemas arrecifales y el manglar, no aparecen representados en estos resultados.

Arrastres adecuados pudieron haber ayudado a la obtención de una mayor diversidad de especies, considerando que se trata de una zona tropical. Todos los taxones encontrados constituyen registros nuevos para el Parque Nacional Arrecife Puerto Morelos. El resultado más relevante obtenido, aparte de que es la primera contribución al estudio de los mísidos de la zona, es el primer registro de Heteromysis elegans, $H$. floridensis y H. siciliseta, para el Caribe mexicano. Nuevas colectas en la zona, seguramente ofrecerán resultados taxonómicos de gran interés.

\section{AGRADECIMIENTOS}

Al Programa de Apoyo a Proyectos de Investigación e Innovación Tecnológica (UNAM), proyecto PAPIIT-IN220715 por los apoyos financieros otorgados en la colecta científica. A las autoridades de CONAPESCA-DGOPA (SAGARPA) y a la administración del Parque Nacional Arrecife Puerto Progreso, por los permisos otorgados en el ingreso y colecta científica en el parque nacional; así como a la Unidad Académica Puerto Morelos, ICMyL-UNAM por el apoyo en el procesamiento de muestras biológicas colectadas. 


\section{LITERATURA CITADA}

Escobar-Briones, E. 2002. 13. Lophogastrida y Mysida, En Biodiversidad, Taxonomía y Biogeografía de artrópodos de México, Hacia una síntesis de su conocimiento. Llorente Pousquets, J. y J. J. Morrone (editores), III, 690 pp.

Heard, R. W. y W. Price. 2006. Revision of Bowmaniella sensu Bacescu, 1968 (Crustacea: Mysida: Mysidae: Gastrosaccinae): a taxonomic conundrum. Zootaxa, 1269: 1-29.

Markham, J. C., F. E. Donath-Hernández, J. L. Villalobos y A. C. Díaz- Barriga. 1990. Notes on the shallow-water marine crustacea of the caribbean coast of Quintana Roo, Mexico. Anales Inst. Biol. Univ. Nac. Auton. Mexico, Ser. Zool., 61(3): 405-446.

Price, W. W. y R. W. Heard. 2009. Mysida (Crustacea) of the Gulf of Mexico. In: Felder y Camp (editors), Gulf of Mexico, origins, waters and biota. Biodiversity 1: 929-938.

Winfield, I., M. Ortiz, S. Cházaro-Olvera, M. Lozano-Aburto y H. Barrera-Escorcia. 2013. Peracáridos marinos bentónicos (Amphipoda, Cumacea, Isopoda y Tanaidacea), Manual de Laboratorio y Campo. México, Ed. UNAM-FESIZTACALA, 103 pp.

Wittmann, K. J., A. Arani y J. P. Lagardere. 2014. Orders Lophogastrida Boas, 1883, Stygiomysida Tchindonova, 1981 and Mysida Boas, 1883 (also known collectively as Mysidacea) In Treatise on Zoology-Anatomy, Taxonomy, Biology, The Crustacea, Traitè de Zoologie, Vaupel Klein, J. C:, M. Charmantier-Daures y F. R. Schram, (editores) 4B, 396pp. Koninklijke Brill NV.

WoRMS. 2017. World Register of Marine Species. Available from http://www.marinespecies.org at VLIZ. Accessed 2017-01-24. doi:10.14284/170. 